Industrial Health, 1977, 15, 175.

\title{
SPECIFIC AND NONSPECIFIC HEMOLYSIS INDUCED BY MERCURIALS AND OTHER HEMOLYSINS
}

During the course of our experimental studies on methylmercury toxicity, the hemolytic effect on erythrocytes was examined in comparison with other mercurials. We have already reported that the hemolytic potency of mercurials was the following descending order: $\mathrm{HgCl}_{2}>p$-chloromercuribenzoate $(\mathrm{PCMB})>$ methylmercuric chloride $(\mathrm{MMC})^{1)}$. Additionally, all these mercurials were shown to increase the osmotic fragility of erythrocytes in hypotonic media. In this preliminary report, it was intended to determine whether the mercurial-induced hemolysis is specific or nonspecific by the use of the simple method presented by Seeman ${ }^{2}$. A variety of other hemolysins were also tested for their effects on erythrocytes against hypotonic shock in confirmation of the Seeman's report ${ }^{2)}$.

Human blood was drawn from a healthy volunteer and centrifuged to separate off the plasma and buffy coat. The erythrocytes were washed with isotonic saline and suspended to make $20 \%$ hematocrit. Aliquots of $0.1 \mathrm{ml}$ were pipetted from this suspension into $4.9 \mathrm{ml}$ of hypotonic test solution which contained $60 \mathrm{mM} \mathrm{NaCl}, 3.7 \mathrm{mM} \mathrm{Na} 2 \mathrm{HPO}_{4}$, $0.6 \mathrm{mM} \mathrm{NaH} \mathrm{PO}_{4}$ and a test compound in varying concentrations. After incubation for $10 \mathrm{~min}$ at $37^{\circ} \mathrm{C}$, the unhemolyzed erythrocytes were centrifuged down in a refrigerated centrifuge at $10,000 \mathrm{rpm}$ for $1 \mathrm{~min}$. Percent hemolysis was measured by determining hemoglobin in the supernatant colorimetrically at $540 \mathrm{~nm}$ in a spectrophotometer.

The results shown in the left half of Fig. 1 demonstrate that inorganic mercury at $0.15-15 \mu \mathrm{M}$ protects erythrocytes against hypotonic hemolysis in addition to its lytic action at higher concentrations, while organic mercurials such as PCMB and MMC proved to be only lytic. Since the time courses of mercurial hemolysis were characterized by a lag period and slowness, no complete hemolysis was observed within $10 \mathrm{~min}$ even at higher concentrations. The findings concerning the dual action of $\mathrm{Hg}^{2+}$ essentially agreed with the results reported by Jung ${ }^{3)}$. The graphs depicted in the right half of Fig. 1 present the specific hemolysis with digitonin and the nonspecific hemolysis with saponin or lysolecithin.

Seeman ${ }^{2}$ suggested that specific or nonspecific hemolysis refers to whether or not there is a receptor or a component on the erythrocyte membrane which strongly interacts or binds with the hemolysin. He showed cholesterol-extracting agents such as digitonin and saponin as the typical specific hemolysins. In the present experiment, however, saponin was clearly shown to stabilize the erythrocyte membrane at subhemolytic concentrations. Even according to the view of $S_{\text {Seeman }}^{2)}$ who indicated that nonspecific hemolysins are in common surface active and 10-100 times less potent than the specific agents, it might be reasonable to consider saponin as a nonspecific agent. All other detergents (Triton $\mathrm{X}-100$, sod. lauryl sulfate, sod. oleate, sod. deoxycholate, etc.) were also confirmed to be nonspecific hemolysins. 
Y. OHMIYA AND K. KOGA
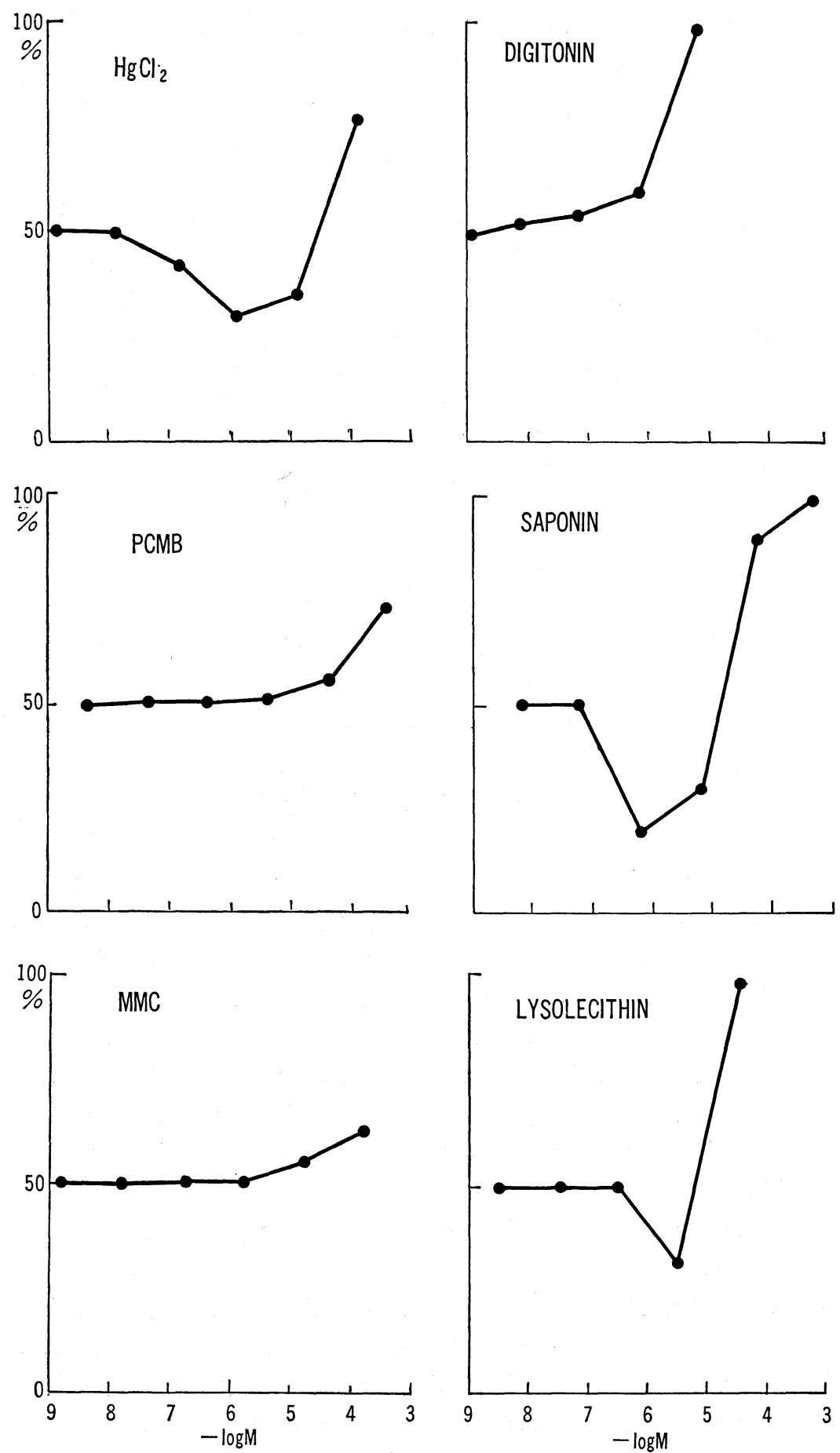

Fig. 1. Hemolysis of human erythrocytes in hypotonic media. The ordinate represents percent hemolysis and the abscissa the molar concentrations of test compounds. Each point represents the mean of 5 or 6 determinations and standard error of the means is within $\pm 10 \%$. 


\section{SPECIFIC AND NONSPECIFIC HEMOLYSIS}

The antihemolytic action of membrane active agents has been explained by an expansion of the erythrocyte membrane followed by an increased critical hemolytic

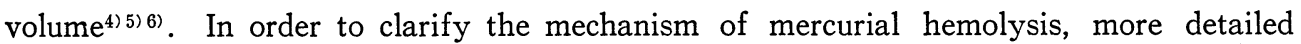
studies including the morphological observations well associated with the kinetics of interaction of mercurials with erythrocytes will be required.

\section{REFERENCES}

1) Ohmiya, Y. (1977). J. Toxicol. Sci., 2, 129.

2) Seeman, P. (1966). Biochem. Pharmacol., 15, 1767.

3) Jung, F. (1947). Arch. Exp. Pathol. Pharmakol., 204, 139.

4) Seeman, P., Kwant, W.O., Sauks, T. and Argent, W. (1969). Biochim. Biophys. Acta, 183, 490.

5) Sheppard, H., Tien, W.H. and Burghardt, C. (1969). Biochem. Pharmacol., 18, 2215.

6) van Steveninck, J., Gjösund, W.K. and Booij, H.L. (1967). Biochem. Pharmacol., 16, 837.

Department of Pharmacology

Akita University School of Medicine

Hondoh 1-chome, Akita 010 Japan and

Yoshio OHMIYA

Department of Pharmacology

Sapporo Medical College

S. 1., W. 17., Chuo-ku

Sapporo 060 Japan

Kenichi KOGA

(Received November 25, 1977) 